\title{
Binding targets' responses to distractors' locations: Distractor response bindings in a location-priming task
}

\author{
Christian Frings and BirTe Möller \\ Saarland University, Saarbrücken, Germany
}

\begin{abstract}
Responses to target stimuli can be encoded together with distracting objects accompanying these targets into a single stimulus-response episode or a single event file. Repeating any object of such an episode can trigger the response encoded in this episode. Hence, repeating a distractor may retrieve the response given to the target that was accompanied by this distractor. In the present experiments, we analyzed whether the binding of target responses to the distractor can be generalized even to the location of a distractor. In two experiments, we used a location-based prime-probe task and found that repeating the location of a distractor triggered the response to the target that had previously been accompanied by a distractor in the repeated location, even if the identity of the distractor changed from the prime to the probe.
\end{abstract}

Stimulus features and response features are integrated for creating cognitive representations of objects and events - a process labeled binding (Hommel, 1998; Kahneman \& Treisman, 1984). Object files and event files are stored in memory and are automatically retrieved by subsequently encountered stimuli that match features of the previous episode. This episodic retrieval process is a core feature of automatization in perception and action (Logan, 1988; Treisman, 1992). Retrieving a previous episode can facilitate perception and action in the case of a complete match between the previous and current episodes, but it can also impair the perception of novel stimuli and interfere with responding if the previous episode contains a mismatch with some of the features or response requirements of the current situation (Denkinger \& Koutstaal, 2009; Hommel, 1998, 2004; Pösse, Waszak, \& Hommel, 2006; Treisman, 1992; Waszak, Hommel, \& Allport, 2003, 2005).

Interestingly, binding and retrieval processes not only incorporate those stimulus features that are relevant for determining a response, but also can include information that is irrelevant or even distracting for the current and/ or previous episode (Rothermund, Wentura, \& De Houwer, 2005; see also Frings, in press; Frings, Rothermund, \& Wentura, 2007; Hommel, 1998, 2005, 2007; Mayr \& Buchner, 2006; Mayr, Buchner, \& Dentale, 2009). For example, Rothermund et al. found that a target stimulus retrieves the response that was given at the last instance at which this stimulus was encountered, even if the stimulus feature to which one has to respond was irrelevant in the former encounter. In particular, participants categorized two different stimulus dimensions, and the dimension was switched between two consecutive displays. Rothermund et al. showed that even the irrelevant stimulus dimension on trial $n-1$ retrieved the response given on trial $n-1$ if this feature was repeated as the target dimension in the next display. Performance depended clearly on whether the retrieved response was compatible or incompatible with the to-be-executed response. Such findings attest to the generality of basic binding and retrieval mechanisms in perception and action.

Turning to distractor processing, situations in which the stimulus to which one responds (i.e., the target) is accompanied by an irrelevant stimulus (i.e., the distractor) become particularly interesting. For example, the multipleintegration model of event file creation (e.g., Hommel, 2005) suggests that a stimulus that shares some features with the target or is relevant/salient for other reasons may be bound into the event file including the executed response. Frings et al. (2007) expanded the basic ideas of event file creation (see Hommel, Müsseler, Aschersleben, \& Prinz, 2001, for a framework theory of event coding) to selection situations in which a distractor (sharing the stimulus-response [S-R] mapping with the target) competes with the target for response generation. They argued that the distractor object in such settings becomes part of the whole S-R episode and, further, that repeating the distractor will result in retrieving the whole episode, including the response. Such an effect of distractor-response binding (DRB) could be analyzed in prime-probe tasks by presenting targets and distractors on every display while also orthogonally varying response repetitions and distractor repetitions. In fact, for such tasks, DRB effects would emerge as an interaction of response and distractor

C. Frings, c.frings@mx.uni-saarland.de 
repetitions: Repeating a distractor retrieves the last episode containing that stimulus (i.e., the prime), which also includes the response to the target that was encountered together with this distractor. Such distractor-to-distractor repetitions should lead to the retrieval of an incompatible response in the case of a response change but to a compatible response in the case of a response repetition. Accordingly, in displays using a flanker configuration of target and distractors (i.e., a central target flanked by two identical distractors; e.g., DFD, with F being the target), Frings et al. observed that distractor repetition effects were modulated by response repetition such that distractor repetitions in the case of response repetition facilitated responses, whereas distractor repetitions in the case of response changes decreased performance.

The present article is concerned with analyzing the interaction of response repetitions and distractor repetitions in a location-based prime-probe task in which distractors compete with targets for response execution. In particular, in previous experiments, the DRB effect has been exclusively analyzed in tasks in which participants had to react to the identity or the color of stimuli (Frings, in press; Frings et al., 2007; Mayr \& Buchner, 2006; Rothermund et al., 2005). Here, we explore whether DRB effects can be generalized to distractor locations. Note that this idea is by no means a trivial matter. In fact, we argue that the response to the target becomes bound to the location of another, irrelevant object. In turn, repeating even another object at the former distractor location should retrieve the response to the former target location.

Looking at the literature on feature-response bindings, one might expect to find the DRB effect also for locations. Typically, it has been assumed that spatial information is used or needed to bind features into objects (Kahneman, Treisman, \& Gibbs, 1992), although recent evidence has suggested that spatial information may play a dominant role only for the encoding process, but not for retrieving event or object files from long-term memory (Hommel \& Colzato, 2009). Moreover, there is evidence for spatially unmediated bindings of nonspatial features; for example, features such as the shape of an object can be bound to a response, irrespective of the object's location (e.g., Hommel, 1998). Then again, it is clear that the integration of spatial information in event files is particularly strong if the response set is spatially defined (see Hommel, 2007). In sum, if we assume that the location of a distractor is just another feature of the distractor representation and, additionally, that the location of a distractor is particularly salient in a location-priming task, we can hypothesize that DRB effects emerge also for locations.

With Experiment 1, we transferred the DRB experiments of Frings et al. (2007) to a spatial task. Participants responded to the location of the target by pressing a spatially corresponding key while ignoring the location of the distractor. We orthogonally varied response repetitions and distractor repetitions. Yet one further noteworthy aspect was that we changed the target-defining feature (color) from primes to probes on half of the trials; that is, a color cue before each trial signaled whether the target would be red or green in the next display. In turn, we could analyze location-based DRB effects completely free of identity-based effects; for example, on half of the response repetition trials, two different stimuli (albeit on the same location in the prime and the probe) were used. Note that a DRB effect would be evidenced by the two-way interaction of response relation and distractor relation; that is, the effect of a distractor location repetition should be modulated by whether the response repeats from the prime to the probe or not. In particular, repeated distractor locations should lead to larger benefits when the response repeats from the prime to the probe, as compared with distractor location repetitions with response changes. The three-way interaction of response relation, distractor relation, and target color relation would show whether DRB effects in a spatial-priming task are affected by stimulus identity (color).

\section{EXPERIMENT 1}

\section{Method}

Participants. Thirty students ( 25 women) from Saarland University participated in the experiment; they were paid $€ 4$ for participation. The median age was 23 years, with a range from 19 to 41 years. None of the participants reported any impairment in color vision.

Design. Essentially, the design comprised two within-subjects factors - namely, response relation (repetition vs. change) and distractor relation (repetition vs. change). In addition, the color of the prime target (red vs. green) and the color of the probe target (red vs. green) were varied orthogonally to all other factors. The two color variables were combined into a target color relation (prime-probe target color repeated vs. target color changed) factor.

Materials. The experiment was conducted using the E-Prime software (E-Prime 1.1). Instructions and the fixation marker were shown in white on a black background on a standard CRT screen (refresh rate was $75 \mathrm{~Hz}$ ). The viewing distance was approximately $60 \mathrm{~cm}$. The stimuli were red and green circular dots with a visual angle of $0.53^{\circ}$. They could appear at four different locations on the screen. Underneath every one of these positions, a white line with a horizontal visual angle of $0.95^{\circ}$ marked the location. This set of white lines was centered on the screen. The approximate horizontal visual angle was $6.39^{\circ}$ between the two upper outside positions and $3.15^{\circ}$ between the two lower inside positions, measured between the inner ends of the position markers, respectively. The vertical component of the visual angle between the upper outside and lower inside positions was approximately $0.95^{\circ}$. Each prime and probe display was presented for $150 \mathrm{msec}$ and was preceded by a colored $0.48^{\circ} \times 0.48^{\circ}$ square that served as a cue and was shown for $400 \mathrm{msec}$.

Procedure. The participants were tested individually in soundproof chambers. Instructions were given on the screen and summarized by the experimenter. The participants were instructed to place the middle and index fingers of both hands on the $\mathrm{D}, \mathrm{C}, \mathrm{M}$, and $\mathrm{K}$ keys of the computer keyboard. The $\mathrm{D}$ key corresponded to the upper left position, the $\mathrm{C}$ key to the lower left position, the $\mathrm{M}$ key to the lower right position, and the K key to the upper right position. In each prime and each probe display, one red and one green dot (one marker of the target and one of the distractor position) were presented together, with the four white lines indicating all possible locations for the stimuli. A 400-msec red or green square, presented before stimuli onset, indicated the color that would mark the target position in the following display. For example, if the square was red, in the following display a red dot appeared at the target position and a green dot at the distractor position. The participants' task was to identify the target location by pressing the spatially corresponding key as quickly and correctly as possible. Distractor locations had to be ignored. A single prime-probe sequence included the following 


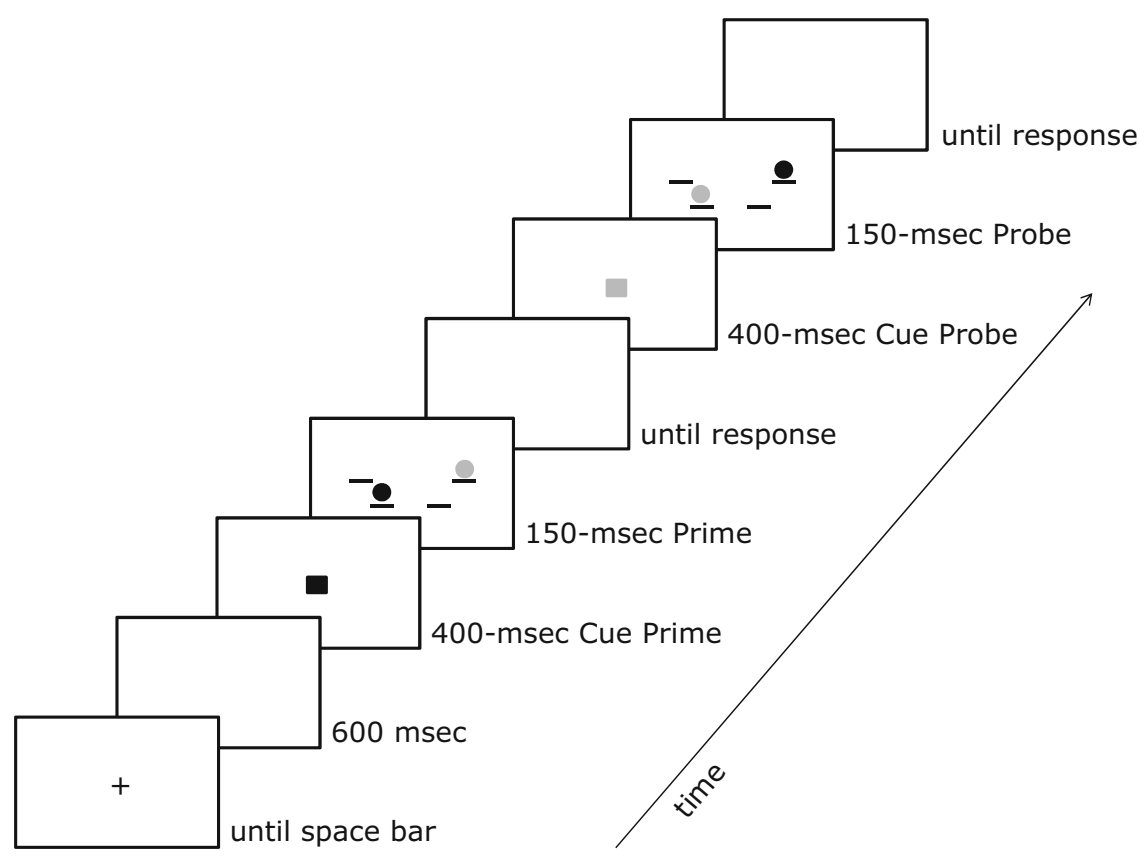

Figure 1. Sequence of events in Experiments 1 and 2. A cue presented before each prime or probe display indicated the target color for the next display. Participants reacted to the location of the target by pressing a spatially corresponding key. Red is depicted in black, whereas green is depicted in gray.

events (see Figure 1). The participant started the sequence by pressing the space bar. After a $600-\mathrm{msec}$ presentation of a blank screen, a red or green cue appeared in the middle of the screen for $400 \mathrm{msec}$, indicating the color with which the target location of the following display would be marked. Then the prime stimulus set was presented for $150 \mathrm{msec}$, consisting of a green and a red dot at two of the possible locations and the four location markers. The participants reacted to the prime target location by pressing the corresponding key. After the response to the prime, a blank screen followed for $100 \mathrm{msec}$ before a $400-\mathrm{msec}$ red or green cue indicated the color that would mark the probe target position. A red and a green dot marking the target and distractor locations of the probe then appeared for $150 \mathrm{msec}$, together with the four location markers. After the response to the probe, a blank screen was presented for $100 \mathrm{msec}$. Finally, a plus sign appeared in the middle of the screen, signaling to the participant that the next trial could be started.

On response repetition (RR) trials, the same position was marked as the target location in the prime and the probe, respectively. On response change $(\mathrm{RC})$ trials, the target position varied between the prime and the probe. Orthogonally to the response relation, the distractor relation was varied. On distractor repetition (DR) trials, the position marked as the distractor location was the same in the prime and the probe, whereas on distractor change (DC) trials, different positions were marked as distractor locations in the prime and the probe. In turn, four different conditions were conducted. On RRDR trials, the prime target and the prime distractor positions were repeated in the probe. On RRDC trials, the prime target position was again marked as the probe target position, whereas the distractor position changed from prime to probe. On RCDR trials, the probe target position differed from the prime target position, whereas the prime distractor position was repeated as the probe distractor position. Finally, on RCDC trials, no position was repeatedly marked from the prime to the probe.

Target positions were marked with a red dot on half of the trials and with a green dot on the other half. This holds true for primes and probes. With the orthogonal variation of response repetition, distractor repetition, target color of the probe, and target color of the prime, 16 different combinations resulted. For every one of these 16 combinations, each of the four possible probe positions was marked as the target location three times (once with each of the remaining three probe positions marked as the distractor location). Prime target and distractor were then randomly assigned to the two other locations. Due to this procedure, 192 different trials resulted. On each particular trial, the prime target and/or the distractor were possibly changed to realize the randomly chosen condition for this trial. For example, on an RRDC trial, the prime target location was changed to the probe target location. During the experimental block, every participant saw all of the 192 trials twice in a random order. An experimental session consisted of a practice block with 32 primeprobe sequences and an experimental block with 384 prime-probe sequences. The four trial types (RRDR, RRDC, RCDR, and RCDC) were realized on 96 trials each.

\section{Results}

Only trials with correct answers to the prime and the probe were considered. Reaction times (RTs) that were more than 1.5 interquartile ranges above the third quartile of the RT distribution of the whole sample (Tukey, 1977) and those that were shorter than $200 \mathrm{msec}$ were excluded from the analysis. Due to these constraints, $15.76 \%$ of all the trials were discarded (probe error rate was $7.25 \%$, prime error rate was $5.64 \%$ ). Mean RTs and error rates for probe displays are given in Table 1.

In a 2 (response relation: RR vs. RC) $\times 2$ (distractor relation: DR vs. DC) $\times 2$ (target color relation in prime and probe: same vs. different) MANOVA with Pillai's trace as the criterion, all main effects of response relation, distractor relation, and target color relation were significant $\left[F(1,29)=4.06, p=.05, \eta_{\mathrm{p}}^{2}=.12\right.$, for response relation; $F(1,29)=25.69, p<.0001, \eta_{\mathrm{p}}^{2}=.47$, for distractor relation; and $F(1,29)=51.81, p<.001, \eta_{\mathrm{p}}^{2}=.64$, for 
Table 1

Mean Reaction Times (RTs, in Milliseconds)

and Mean Error Rates (ERs, in Percentages) As a

Function of Response Relation, Distractor Relation,

and Target Color (Repeated vs. Changed), Experiment 1

\begin{tabular}{|c|c|c|c|c|}
\hline & \multicolumn{4}{|c|}{ Response Relation } \\
\hline & \multicolumn{2}{|c|}{$\begin{array}{l}\text { Response } \\
\text { Repetition }\end{array}$} & \multicolumn{2}{|c|}{$\begin{array}{c}\text { Response } \\
\text { Change }\end{array}$} \\
\hline & RT & ER & RT & ER \\
\hline \multicolumn{5}{|l|}{ Target Color Repeated } \\
\hline Distractor change & 441 & 6.2 & 469 & 8.4 \\
\hline Distractor repetition & 427 & 4.3 & 467 & 8.5 \\
\hline Priming effect $t^{\mathrm{a}}$ & \multicolumn{2}{|c|}{$+14, S E M=4$} & \multicolumn{2}{|c|}{$+2, S E M=3$} \\
\hline \multicolumn{5}{|l|}{ Target Color Changed } \\
\hline Distractor change & 496 & 7.8 & 474 & 8.4 \\
\hline Distractor repetition & 484 & 7.6 & 471 & 6.7 \\
\hline Priming effect ${ }^{\mathrm{a}}$ & \multicolumn{2}{|c|}{$+12, S E M=3$} & \multicolumn{2}{|c|}{$+3, S E M=3$} \\
\hline
\end{tabular}

aPriming effect is computed as the difference between distractor change and distractor repetition.

target color relation, respectively]. Reactions were faster if the target color was repeated from the prime to the probe (451 msec) than if participants had to react to different colors in the prime and probe $(481 \mathrm{msec})$. Also, reactions to repeated target locations $(461 \mathrm{msec})$ were faster than reactions to unrepeated target locations (470 $\mathrm{msec})$, and reactions to displays with repeated distractor positions (461 msec) were faster than reactions to displays with unrepeated distractor positions (469 $\mathrm{msec})$. Importantly, the interaction of response relation and distractor relation was also significant $\left[F(1,29)=15.45, p<.001, \eta_{\mathrm{p}}^{2}=.35\right]$. Moreover, the three-way interaction was not significant $(F<1, p=.76)$, indicating that changes in the target color between the prime and probe did not moderate the interaction of response relation and distractor relation. In fact, for trials with no color changes between the prime and probe, the interaction of distractor relation and response relation was significant $[F(1,29)=5.01, p=.03$, $\left.\eta_{\mathrm{p}}^{2}=.15\right]$, as well as for trials with target color changes $\left[F(1,29)=6.63, p=.02, \eta_{\mathrm{p}}^{2}=.19\right]$.

For the sake of completeness, the interaction of target color relation and response relation was also significant $\left[F(1,29)=86.66, p<.001, \eta_{\mathrm{p}}^{2}=.75\right]$, indicating different RTs for target color relation (same vs. different) if the response repeated from the prime to probe. The interaction of distractor relation and target color relation did not reach significance $\left[F(1,29)<1, p=.85, \eta_{\mathrm{p}}^{2}=.001\right]$.

A MANOVA on error rates, using the same design, revealed significant main effects $[F(1,29)=6.10, p=$ $.02, \eta_{\mathrm{p}}^{2}=.17$, and $F(1,29)=6.0, p=.02, \eta_{\mathrm{p}}^{2}=.17$, for response relation and distractor relation, respectively], indicating fewer errors for both response repetition and distractor repetition. The main effect of target color relation was not significant $\left[F(1,29)=3.20, p=.08, \eta_{\mathrm{p}}^{2}=\right.$ .10]. A significant interaction of target color relation and response relation $\left[F(1,29)=9.05, p=.01, \eta_{\mathrm{p}}^{2}=.24\right]$ indicated exceptionally few errors in response repetition trials if the target color was the same in the prime and probe. Neither the interactions of target color relation and distractor relation $\left[F(1,29)<1, p=.97, \eta_{\mathrm{p}}^{2}<.001\right]$ and response relation and distractor relation $[F(1,29)<1, p=$ $\left..71, \eta_{\mathrm{p}}^{2}=.01\right]$ nor the three-way interaction $[F(1,29)=$ $\left.3.86, p=.06, \eta_{\mathrm{p}}^{2}=.12\right]$ reached significance.

\section{Discussion}

We found an interaction of response and distractor repetition and, hence, found DRB effects for distractor locations in a location-based prime-probe design. This finding effectively expands previous DRB effects with identity (e.g., Frings et al., 2007) to spatial information and, in turn, confirms that DRB is a general phenomenon. In addition, one might argue that the interaction of response and distractor repetition in identity tasks hinges on the RRDR condition - that is, on a condition in which a complete match between the prime and probe exists (e.g., Frings, in press; although see Experiment 2 in Frings et al., 2007, for an exception). Here, on trials on which the target-defining feature changed between the prime and probe, participants faced displays in the RRDR condition that were, in fact, only a partial match (i.e., the same stimuli were repeated but at opposite locations). Yet the modulation of distractor-to-distractor repetition effects by response repetition was not influenced by changing the target-defining feature. In turn, the DRB effects could not be explained, due to a complete repetition of the prime display as the probe display in the RRDR condition. However, it should be acknowledged that we used only two colors, and hence, a target color change from prime to probe always implied a distractor color change from prime to probe. Any effect of target identity on the spatial DRB effect could also have been an effect of distractor identity (or a combination of both).

Note that response repetition effects were significantly modulated by target color repetitions; in fact, repeated responses were slower when the target changed the color from prime to probe. This is the typical pattern one observes in such tasks (see, e.g., Milliken, Tipper, Houghton, \& Lupiáñez, 2000). Sometimes, such effects have been explained in terms of feature mismatch (Park \& Kanwisher, 1994; but see Tipper, Weaver, \& Milliken, 1995). In particular, the prime color becomes bound to the prime target location, and when a different color is repeated at the same location, RTs lengthen due to the feature mismatch of color and location. In addition, such effects have been interpreted in terms of inhibition of return (Milliken et al., 2000; we discuss this phenomenon in the General Discussion section). Of course, this data pattern can also be explained in terms of the theory of event coding: Target color changes with location repetitions are only partial matches and, hence, diminish performance (Hommel, 1998, 2004). It should be noted, however, that the crucial interaction of distractor relation and response relation cannot be explained in terms of feature mismatch, since it was unaffected by the target color relation factor.

However, one caveat to the experiment might have been the design that extensively used response repetitions, thereby possibly inviting participants to use strategies based on the contingency. Christie and Klein (2001, 2008) have argued specifically for location-based primeprobe designs to conduct all possible conditions in order to avoid contingency strategies; that is, they argue for using 
distractor-to-target repetitions, target-to-distractor repetitions, and complete switches, in addition to the conditions in Experiment 1 (see Frings \& Wentura, 2006, 2008, for similar arguments on contingency-based distractor processing for identity-based prime-probe tasks). In fact, when one looks at the data in Christie and Klein (2001, first experiment), it seems that they nevertheless obtained the interaction of response repetitions and distractor repetitions. However, we thought it prudent to replicate these findings with Experiment 2 for two reasons. First, Christie and Klein (2001) did not report this crucial interaction, because they were exclusively interested in distractor-to-target repetitions. Second, they did not vary the target-defining feature between primes and probes in their experiment.

\section{EXPERIMENT 2}

\section{Method}

Participants. Thirty-four new students (25 women) from Saarland University participated in the experiment; they were paid $€ 4$ for participation. The median age was 24 years, with a range from 20 to 32 years. None of the participants reported any impairment in color vision.

Design. To the four conditions of Experiment 1, three conditions were added - namely, a target-to-distractor condition, a distractorto-target condition, and a switch condition (location of the prime target becomes location of the probe distractor and vice versa). Again, color of the prime target (red vs. green) and color of the probe target (red vs. green) were varied orthogonally to all other factors. The two color variables were combined into a target color relation (primeprobe target color repeated vs. target color changed) factor.

Materials and Procedure. The materials and procedure were the same as those in Experiment 1, with the following exceptions. In addition to the RRDR, RRDC, RCDR, and RCDC conditions, three new conditions were realized. In the distractor-to-target (DT) condition, the prime distractor position was repeated as the probe target, whereas the probe distractor location was not marked in the prime. In the target-to-distractor (TD) condition, the prime target location was marked in the probe as the distractor with a new probe target position. Finally, in the switch condition, the distractor location of the prime became the target in the probe and the target of the prime was marked as the distractor in the probe.

Target positions were marked with a red dot on half of the trials and with a green dot on the other half. This holds true for primes and probes. With the orthogonal variation of the seven conditions, target color of the probe, and target color of the prime, 28 different combinations resulted. For every one of these 28 combinations, each of the four positions was marked as the probe target location three times (once together with each of the remaining three probe positions marked as the probe distractor). The prime target and distractor were randomly assigned to the two left positions. This procedure resulted in 336 different trials. As in Experiment 1, the locations of the stimuli were changed before presentation to realize the particular condition, according to the current trial type. An experimental session consisted of a practice block with 32 prime-probe sequences and an experimental block with 336 prime-probe sequences. The seven trial types (RRDR, RRDC, RCDR, RCDC, DT, TD, and switch) were realized on 48 trials each.

\section{Results}

Only trials with correct answers to the prime and the probe were considered. RTs that were more than 1.5 interquartile ranges above the third quartile of the RT distribution of the whole sample (Tukey, 1977) and those that were shorter than $200 \mathrm{msec}$ were excluded from the analysis. Due to these constraints, $12.1 \%$ of all the trials were discarded (probe error rate was $4.65 \%$; prime error rate was 3.08\%). Mean RTs of all seven conditions are depicted in Figure 2. Mean RTs and error rates for probe displays corresponding to the conditions in Experiment 1 are depicted in Table 2.

We conducted a MANOVA on the same conditions as those in Experiment 1, with RT as the dependent variable. In a 2 (response relation: RR vs. RC) $\times 2$ (distractor relation: DR vs. DC) $\times 2$ (target color relation in prime and probe: same vs. different) MANOVA with Pillai's trace as criterion, the main effect of response relation was not significant $\left[F(1,33)=3.25, p=.08, \eta_{\mathrm{p}}^{2}=.09\right]$, whereas the main effects of distractor relation and target color relation both reached significance $[F(1,33)=16.30, p<$ $.001, \eta_{\mathrm{p}}^{2}=.33$, and $F(1,33)=99.56, p<.001, \eta_{\mathrm{p}}^{2}=.75$, respectively]. Reactions were faster if the target color was repeated from the prime to the probe $(445 \mathrm{msec})$ than if the participants had to react to different colors in the prime and probe $(485 \mathrm{msec})$. Also, reactions to displays with repeated distractor positions $(459 \mathrm{msec})$ were faster than reactions to displays with unrepeated distractor positions $(468 \mathrm{msec})$. Importantly, the interaction of response relation and distractor relation was also significant $[F(1,33)=$ $\left.5.36, p=.03, \eta_{\mathrm{p}}^{2}=.14\right]$. Again, the three-way interaction was not significant $(F<1, p=.94)$, indicating that target color relation did not moderate the interaction of response relation and distractor relation. In fact, as in Experiment 1, the response relation $\times$ distractor relation interaction was significant if the target color did not change $[F(1,33)=$ 2.94, $p=.05$ (one-tailed), $\left.\eta_{\mathrm{p}}^{2}=.08\right]$, as well as if the target color changed from the prime to the probe display $\left[F(1,33)=3.26, p=.04\right.$ (one-tailed), $\left.\eta_{\mathrm{p}}^{2}=.09\right]$.

As in Experiment 1, the interaction of response relation and target color relation reached significance $[F(1,33)=$ $144.79, p<.001, \eta_{\mathrm{p}}^{2}=.81$ ], indicating different RTs for target color relation (same vs. different) if the response repeated from the prime to the probe. The interaction of distractor relation and target color relation did not reach significance $\left[F(1,33)<1, p=.78, \eta_{\mathrm{p}}^{2}=.002\right]$.

A MANOVA on error rates, using the same design, revealed significant main effects $[F(1,33)=10.09, p=$ $.003, \eta_{\mathrm{p}}^{2}=.23$, and $F(1,33)=15.77, p<.001, \eta_{\mathrm{p}}^{2}=.32$, for distractor relation and target color relation, respectively], indicating fewer errors for both target color repetition and distractor repetition. The main effect of response relation did not reach significance $[F(1,33)<1, p=1$, $\left.\eta_{\mathrm{p}}^{2}<.001\right]$. None of the two-way interactions reached significance $\left[F(1,33)=2.96, p=.1, \eta_{\mathrm{p}}^{2}=.08\right.$, for target color relation $\times$ response relation; $F(1,33)=1.79, p=$ $.19, \eta_{\mathrm{p}}^{2}=.05$, for target color relation $\times$ distractor relation; and $F(1,33)=1.58, p=.22, \eta_{\mathrm{p}}^{2}=.05$, for response relation $\times$ distractor relation, respectively]. The three-way interaction $\left[F(1,33)=3.90, p=.06, \eta_{\mathrm{p}}^{2}=.11\right]$ was not significant either.

\section{Discussion}

As in Experiment 1, we observed a significant interaction of response and distractor repetitions in a locationbased prime-probe task, indicating a DRB effect for the 

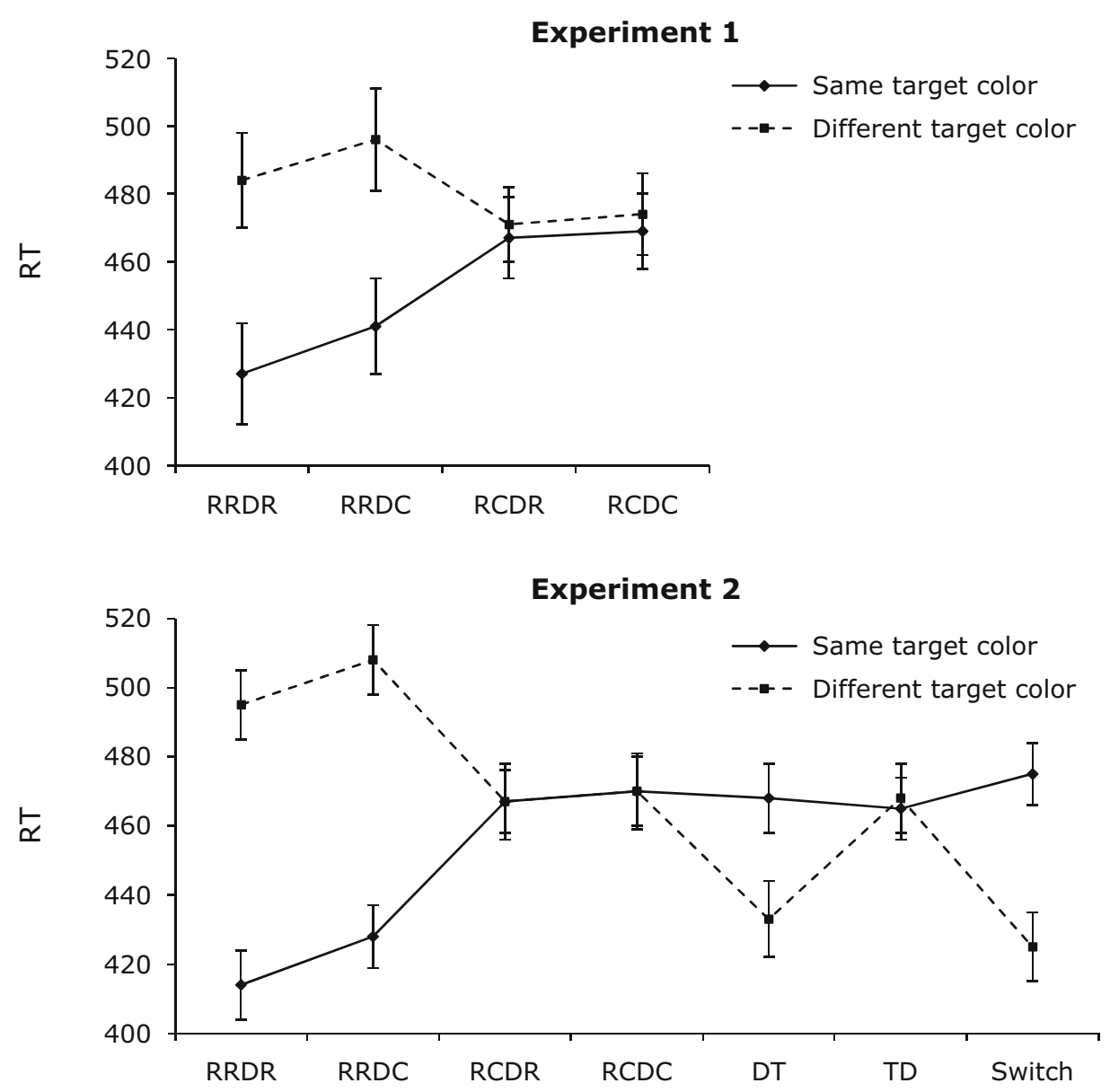

Figure 2. Mean reaction times (RTs, in milliseconds) of all conditions as a function of target color relation of the prime and probe displays, Experiments 1 and 2. Error bars depict standard deviations of the means.

distractor location. In addition, the DRB effect did not hinge on complete repetitions of primes as probes in the RRDR condition, and it also remained significant when participants could not use contingency-based strategies due to a high proportion of response repetitions. ${ }^{1}$

Table 2

Mean Reaction Times (RTs, in Milliseconds) and Mean Error Rates (ERs, in Percentages) As a Function of Response Relation, Distractor Relation, and Target Color (Repeated vs. Changed), Experiment 2

\begin{tabular}{|c|c|c|c|c|}
\hline & \multicolumn{4}{|c|}{ Response Relation } \\
\hline & \multicolumn{2}{|c|}{$\begin{array}{l}\text { Response } \\
\text { Repetition }\end{array}$} & \multicolumn{2}{|c|}{$\begin{array}{c}\text { Response } \\
\text { Change }\end{array}$} \\
\hline & RT & ER & $\mathrm{RT}$ & ER \\
\hline \multicolumn{5}{|l|}{ Target Color Repeated } \\
\hline Distractor change & 428 & 3.9 & 470 & 3.4 \\
\hline Distractor repetition & 414 & 1.7 & 467 & 4.2 \\
\hline Priming effect ${ }^{a}$ & \multicolumn{2}{|c|}{$+14, S E M=5$} & \multicolumn{2}{|c|}{$+3, S E M=4$} \\
\hline \multicolumn{5}{|l|}{ Target Color Changed } \\
\hline Distractor change & 508 & 8.1 & 470 & 7.4 \\
\hline Distractor repetition & 495 & 6.0 & 467 & 4.8 \\
\hline Priming effect ${ }^{\mathrm{a}}$ & \multicolumn{2}{|c|}{$+13, S E M=4$} & \multicolumn{2}{|c|}{$+3, S E M=4$} \\
\hline
\end{tabular}

aPriming effect is computed as the difference between distractor change and distractor repetition.

\section{GENERAL DISCUSSION}

The present experiments were concerned with whether DRB effects could be observed for the location, instead of the identity, of distractors. In fact, in two experiments using a location-based prime-probe paradigm, we found effects of distractor location repetitions to be modulated by response repetitions. In other words, repeating the location of a distractor from the prime to the probe retrieves the response given to the prime target. Thus, as was hypothesized in the introduction, spatial distractor information can be bound into an event file just like any other feature (e.g., color). In addition, DRB effects for location did not hinge on stimulus repetitions. Note that we observed the interaction of response repetition and distractor repetition irrespective of whether the target color (and, in turn, the distractor color as well) changed from the prime to the probe or not. Finally, the proportion of response repetitions and, thereby, possible strategies of the participants did not modulate DRB effects for locations. In sum, the DRB effect could be generalized to spatial information.

Yet it should be noted that Frings et al. (2007) observed a disordinal interaction between distractor and response repetition; that is, repeating distractors in the case of a 
response repetition increased the benefit from response repetition, whereas repeating a distractor in the case of response change led to cost effects. In the present experiments, we replicated the interaction of response repetition and distractor repetition, which showed that distractor repetitions again boosted benefit effects from response repetition, whereas distractor-to-distractor repetitions with response changes led to null effects. Note, however, that the general idea that distractors retrieve responses to previous targets does not depend on negative effects in the condition without response repetition but, instead, exclusively hinges on the interaction effect of response repetition and distractor repetition, which was significant. In particular, only the binding approach predicts that distractor repetitions should be faster when the retrieved response is compatible (see Frings, in press). In contrast, an alternative approach concerning distractor processing is the inhibition account (e.g., Frings \& Wühr, 2007; Houghton \& Tipper, 1994). Such an account would always predict benefits for repeated distractors: It should be easier to process the target and to select the appropriate response if the probe distractor still suffers from the inhibition that it received during the prime. Yet the fact that the size of the distractor repetition benefit varied as a function of the response relation cannot be explained by an inhibition of the distractor. Instead, an inhibition theory would predict equal distractor repetition benefits in all conditions, since the persisting inhibition of a prime distractor is assumed to be independent of the relation between prime and probe response.

Yet one might speculate that both mechanisms can work in parallel. If so, distractor inhibition would further boost the benefit from distractor repetitions on response repetition trials, whereas the cost effect from repeated distractors with response changes might be out-canceled or overshadowed by distractor inhibition. This might explain why, in some experiments, distractor-to-distractor repetitions with response changes led to null effects.

A further phenomenon that might have occurred in our experiments is the inhibition of return (IOR; Posner \& Cohen, 1984). IOR refers to the observation that participants are often slower to detect a target at a previously cued location than at a previously uncued location. Yet IOR-like effects can also occur in spatial-priming tasks as used in our experiments (see Christie \& Klein, 2001; Milliken et al., 2000). In particular, in such tasks, IOR would emerge as slowed responses to probe locations that have been occupied in the prime display if other effects, such as feature mismatches or priming of features, are controlled for (see Milliken et al., 2000). Thus, in our experiments, the conditions with color target changes would be the best option for analyzing IOR. In fact, our results seem to be mixed with respect to IOR-like effects. For response repetitions, we indeed found slower responses, possibly indicating IOR, but for distractor-to-target repetitions, as well as for complete switches of distractors and targets, we did not observe slowed responses. More important, the interaction of distractor repetition and response repetition could not be explained in terms of IOR, because then, for example, the RRDR condition should not be faster than the RRDC condition.
Finally, at least two further issues should be considered here. First, in the present experiments, spatial information was task relevant, since the participants responded to the location of the cued stimulus. Hence, the spatial information of the uncued (i.e., distractor) stimulus might have been primed due to this task relevance as well (see Hommel, 2007). In addition, the distractor location was always a location included in the S-R mapping; that is, the distractor always appeared at a location at which a target could possibly appear. This might have enhanced the salience of the spatial distractor information (because it clearly interfered with responding to the target location; see Frings \& Wentura, 2006), and, in turn, binding was more likely (Hommel, 2005). Likewise, Spapé and Hommel (2008) demonstrated that context modulations of cognitive control processes are due to episodic retrieval; that is, they showed that task-specific control information can be integrated together with the stimuli and actions in an event file. Thus, reactivation of a part of such an episode can also retrieve some form of executive control. Further evidence comes from Experiment 1B in Frings et al. (2007), in which the positions of the targets and distractors were orthogonally varied to identity. Participants had to respond to the targets' identities, and the interaction of response and distractor repetition emerged. Yet, in the DRB, effect for identity was not significantly modulated by the location of targets or distractors. In concert, these findings suggest that for bindings of target responses to distractor locations, spatial information must be task relevant, as has been shown for other aspects of stimulus representation previously.

The second issue worth discussing is the targetdistractor distance. In our experiments, distractors and targets were always presented with only a marginal distance between them. Yet, obviously, it would be very inefficient for our cognitive system to bind every distracting stimulus regardless of its distance from the response-relevant stimulus into an event file. In fact, there is evidence for a direct influence of the amount of distance for binding features into object files (see, e.g., Xu, 2006). In addition, the target-distractor distance is known to affect the degree to which a distractor is processed (and in turn, interferes with the target; see, e.g., Ruthruff \& Miller, 1995). On the other hand, it may be not only the distance that modulates whether a distractor and a target are bound together, but also the way in which we perceive them as belonging to the same object or not. In particular, Frings and Rothermund (2010) found DRB effects for identities to be modulated by such grouping principles irrespective of the spatial distance. Yet, for the experiments presented here, it can be concluded that with a marginal distance between targets and distractors, a spatial DRB effect can be found.

\section{AUTHOR NOTE}

The research reported in this article was supported by a grant from the Deutsche Forschungsgemeinschaft to C.F. (FR 2133/1-1). We are grateful to John Christie, Michiel Spapé, Yaffa Yeshurun, and an anonymous reviewer for their helpful comments on an earlier version of the manuscript. In addition, we thank Charlotte Schwedes, Michaela Wanke, and Manfred Neumann for conducting the experiments. Correspondence concerning this article may be addressed to C. Frings, Faculty of Behavioral Sciences, Department of Psychology, Saarland 
University, Campus A 2.4, D-66123 Saarbrücken, Germany (e-mail: c.frings@mx.uni-saarland.de).

\section{REFERENCES}

Christie, J.[J.], \& KLeIN, R. M. (2001). Negative priming for spatial location? Canadian Journal of Experimental Psychology, 55, 24-38.

Christie, J. J., \& KLein, R. M. (2008). On finding negative priming from distractors. Psychonomic Bulletin \& Review, 15, 866-873.

Denkinger, B., \& Koutstaal, W. (2009). Perceive-decide-act, perceive-decide-act: How abstract is repetition-related decision learning? Journal of Experimental Psychology: Learning, Memory, \& Cognition, 35, 742-756.

Frings, C. (in press). On the decay of distractor-response episodes. Experimental Psychology.

Frings, C., \& Rothermund, K. (2010). To be, or not to be . . included in an event file: When are distractors integrated into $S-R$ episodes and used for response retrieval? Manuscript submitted for publication.

Frings, C., Rothermund, K., \& Wentura, D. (2007). Distractor repetitions retrieve previous responses to targets. Quarterly Journal of Experimental Psychology, 60, 1367-1377.

Frings, C., \& Wentura, D. (2006). Strategy effects counteract distractor inhibition: Negative priming with constantly absent probe distractors. Journal of Experimental Psychology: Human Perception \& Performance, 32, 854-864.

Frings, C., \& Wentura, D. (2008). Separating context and trial-by-trial effects in the negative priming paradigm. European Journal of Cognitive Psychology, 20, 195-210.

FrINGS, C., \& WüHR, P. (2007). On distractor repetition benefits in the negative-priming paradigm. Visual Cognition, 15, 166-178.

Hommel, B. (1998). Event files: Evidence for automatic integration of stimulus-response episodes. Visual Cognition, 5, 183-216.

Hommel, B. (2004). Event files: Feature binding in and across perception and action. Trends in Cognitive Sciences, 8, 494-500.

Hommel, B. (2005). How much attention does an event file need? Journal of Experimental Psychology: Human Perception \& Performance, 31, 1067-1082.

Hommel, B. (2007). Feature integration across perception and action: Event files affect response choice. Psychological Research, 71, 42-63.

Hommel, B., \& Colzato, L. S. (2009). When an object is more than a binding of its features: Evidence for two mechanisms of visual feature integration. Visual Cognition, 17, 120-140.

Hommel, B., Müsseler, J., Aschersleben, G., \& Prinz, W. (2001). The theory of event coding (TEC): A framework for perception and action planning. Behavioral \& Brain Sciences, 24, 849-937.

Houghton, G., \& Tipper, S. P. (1994). A model of inhibitory mechanisms in selective attention. In D. Dagenbach \& T. H. Carr (Eds.), Inhibitory processes in attention, memory, and language (pp. 53-112). San Diego: Academic Press.

Kahneman, D., \& Treisman, A. (1984). Changing views of attention and automaticity. In R. Parasuraman \& R. Davies (Eds.), Varieties of attention (pp. 29-61). Orlando, FL: Academic Press.

Kahneman, D., \& Treisman, A., \& Gibbs, B. J. (1992). The reviewing of object files: Object-specific integration of information. Cognitive Psychology, 24, 175-219.

LogAn, G. D. (1988). Toward an instance theory of automatization. Psychological Review, 95, 492-527.

Mayr, S., \& Buchner, A. (2006). Evidence for episodic retrieval of inadequate prime responses in auditory negative priming. Journal of
Experimental Psychology: Human Perception \& Performance, 32, 932-943

Mayr, S., Buchner, A., \& Dentale, S. (2009). Prime retrieval of motor responses in negative priming. Journal of Experimental Psychology: Human Perception \& Performance, 2, 408-423.

Milliken, B., Tipper, S. P., Houghton, G., \& Lupiáñez, J. (2000). Attending, ignoring, and repetition: On the relation between negative priming and inhibition of return. Perception \& Psychophysics, 62, 1280-1296.

PARK, J., \& KANWISHER, N. (1994). Negative priming for spatial location: Identity mismatching, not distractor inhibition. Journal of Experimental Psychology: Human Perception \& Performance, 20, 613-623.

Posner, M. I., \& Cohen, Y. (1984). Components of visual orienting. In H. Bouma \& D. G. Bouwhuis (Eds.), Attention and performance X: Control of language processes (pp. 551-556). Hillsdale, NJ: Erlbaum.

Pösse, B., Waszak, F., \& Hommel, B. (2006). Do stimulus-response bindings survive a task switch? European Journal of Cognitive Psychology, 18, 640-651.

Rothermund, K., Wentura, D., \& De Houwer, J. (2005). Retrieval of incidental stimulus-response associations as a source of negative priming. Journal of Experimental Psychology: Learning, Memory, \& Cognition, 31, 482-495.

Ruthruff, E., \& Miller, J. (1995). Negative priming depends on ease of selection. Perception \& Psychophysics, 57, 715-723.

Spapé, M. M., \& Hommel, B. (2008). He said, she said: Episodic retrieval induces conflict adaptation in an auditory Stroop task. Psychonomic Bulletin \& Review, 15, 1117-1121.

Tipper, S. P., Weaver, B., \& Milliken, B. (1995). Spatial negative priming without mismatching: Comment on Park and Kanwisher. Journal of Experimental Psychology: Human Perception \& Performance, 21, 1220-1229.

Treisman, A. (1992). Perceiving and re-perceiving objects. American Psychologist, 47, 862-875.

TukeY, J. (1977). Exploratory data analysis. Reading, MA: AddisonWesley.

WaszaK, F., Hommel, B., \& Allport, A. (2003). Task-switching and long-term priming: Role of episodic stimulus-task bindings in taskshift costs. Cognitive Psychology, 46, 361-413.

WaszaK, F., Hommel, B., \& Allport, A. (2005). Interaction of task readiness and automatic retrieval in task switching: Negative priming and competitor priming. Memory \& Cognition, 33, 595-610.

XU, Y. (2006). Understanding the object benefit in visual short-term memory: The roles of feature proximity and connectedness. Perception \& Psychophysics, 68, 815-828.

\section{NOTE}

1. In fact, even in Experiment 2, target and distractor location repetitions occurred slightly above chance [two sevenths $(\sim 28.5 \%)$ of the time, as compared with the one quarter $(25 \%)$ of the time one would expect in a fully balanced design]. Thus, it may still be possible that retrieval processes are slightly enhanced in the context of this experiment, due to the repetition bias.

(Manuscript received March 15, 2010; revision accepted for publication June 17, 2010.) 